\title{
Enseñanza de las Estrategias de Comprensión del Lenguaje Escrito: Selección y secuenciación de objetivos y contenidos
}

\author{
José Escoriza Nieto
}

Departamento de Psicología Evolutiva y de la Educación, Universidad de Barcelona

\section{España}




\section{Resumen}

La elaboración de este artículo ha perseguido un doble objetivo. Por una parte, proceder al análisis de la problemática relativa a la enseñanza del conocimiento estratégico, articulada en torno a la toma de decisiones didácticas relacionadas con tres de las cuatro cuestiones básicas a las que todo tipo de diseño curricular debe dar una respuesta adecuada: ¿por qué, qué y cuándo enseñar? Teniendo en cuenta esta finalidad, se ha elaborado una propuesta en la que se concretan y exponen todas aquellas operaciones cognitivas, cuya ejecución se presupone va a permitir el logro de los diferentes objetivos específicos e instrumentales, con respecto a la comprensión del LE (Selección, Organización, Elaboración/Integración y Expresión/Comunicación del conocimiento) y que están relacionados con la secuencia integrada por las siguientes Acciones Cognitivas: Subrayar, Resumir, Jerarquizar las Ideas en función de su naturaleza inclusora, Relacionar los Conocimientos Previos con los contenidos del discurso escrito y Producir una Composición Escrita. Por otra, proporcionar a los profesionales, que desarrollan su actividad en instituciones educativas, una guía que pueda ser de utilidad tanto para el diseño de procesos de E-A del conocimiento estratégico, como para la evaluación de las dificultades producidas en el proceso de comprensión del LE, en general y, de forma específica, a nivel de aplicación práctica de cada una de las operaciones cognitivas que figuran como componentes de las diversas Acciones Cognitivas seleccionadas como integrantes esenciales de la estructura interna de la Actividad de lectura/escritura.

Palabras Clave/Keywords: Estrategias, Comprensión, Lectura, Escritura, Lenguaje Escrito, Enseñanza, Aprendizaje, Dificultades, Evaluación. 


\section{Introducción}

El diseño y experimentación de programas de intervención orientados a la enseñanza de las Estrategias de Comprensión del lenguaje escrito han venido recibiendo una atención sistemática prácticamente desde los inicios de la década de los 80 . Ello se debió, entre otros factores, al interés de los investigadores por elaborar una teoría cognitiva de la comprensión (Lorch y van den Broek, 1997), sobre todo a raíz de los estudios pioneros realizados en el Center for Study of Reading en la Universidad de Illinois y a la influencia que habría de tener el artículo publicado por Kintsch y van Dijk (1978) acerca de la naturaleza de los procesos que tienen lugar durante la lectura. Algunos de los trabajos pioneros fueron los realizados por Just y Carpenter, 1980; Beck, Omanson y McKeown, 1982; Tharp, 1982; Paris, Lipson y Wilson, 1983; Pearson y Gallagher, 1983, etc. Este interés se fue incrementando de forma progresiva a medida que los estudios realizados iban aportando datos demostrativos de que la aptitud decodificadora, aún siendo valorada como necesaria, no podía ser considerada como una competencia suficiente con respecto al empleo de la lectura como instrumento de aprendizaje. La diversidad de propuestas formuladas han compartido el objetivo de tratar de concretar las estrategias que podrían suponer un mayor nivel de eficacia en la mejora de los procesos de comprensión y, por tanto, las que permitirían definir de una forma más precisa, fiable y válida la competencia estratégica del alumnado. Maria (1987) ha realizado una revisión de esta problemática, articulada en torno a dos de las cuestiones básicas relacionadas con la enseñanza de la comprensión: ¿qué debemos enseñar? y ¿cómo debería ser enseñada? La cuestión de ¿qué enseñar? ha sido objeto de dos planteamientos controvertidos: si los contenidos seleccionados deberían ser habilidades concretas, estrategias individuales, listas o repertorios de estrategias, etc, antes, durante o después de la lectura o, por el contrario, lo que debe ser enseñado es el proceso de comprensión, como un proceso holístico, es decir, de principio a fin y compuesto por una secuencia ordenada de estrategias. En ambos casos, el problema de qué Estrategias seleccionar, como contenidos pertinentes y relevantes, sigue siendo aún objeto de evidentes discrepancias entre los investigadores. Según Pressley (1998), en la evolución de los estudios sobre Estrategias de comprensión podemos diferenciar dos momentos concretos. El primero se caracteriza por la investigación de estrategias individuales, tales como por ejemplo: activación de los conocimientos previos, identificación de las ideas principales, análisis de la estructura de los textos narrativos, resumir, etc. El segundo surge como consecuencia del desarrollo de modelos cognitivos más sofisticados y de los que se derivó la hipótesis de que el proceso 
de comprensión debería ser analizado como una totalidad no segmentable en habilidades o estrategias concretas.

La segunda cuestión que cita Maria (1987) es la que hace referencia a ¿cómo enseñar? Tratar de responder a esta otra pregunta ha dado lugar a una serie de investigaciones centradas en el estudio de la problemática relativa a la formulación/elección de un determinado modelo instruccional y cuyo objetivo básico y general lo podemos concretar en tratar de promover en el alumnado la competencia en la realización de autoaprendizajes: que el alumno/a aprenda a aprender. Con la finalidad de ofrecer una respuesta adecuada a esta cuestión, la autora analiza y describe las características de los tres modelos instruccionales elaborados: modelo basado en las características del texto, modelo de enseñanza explícita y modelo combinado. La elección de uno u otro modelo dependerá de factores diversos, tales como por ejemplo: la interpretación que se defienda con respecto al proceso de lectura, si la comprensión es entendida como un componente de habilidades o si, por el contrario, es conceptualizada como un proceso holístico y el marco teórico seleccionado como relevante, con respecto a la explicación de los procesos de Enseñanza- Aprendizaje (E-A). A partir de la llamada "revolución cognitiva", los dos modelos instruccionales, con mayor tradición e influencia, han sido el denominado como Instrucción Directa (Roehler y Duffy, 1984) o Enseñanza Explícicita (Pearson y Gallagher, 1983) y el de Enseñanza Recíproca, formulado por Palincsar y Brown (1984). Posteriormente, Pressley, El-Dinary, Gaskins et al. (1992) elaboraron una nueva propuesta de intervención descrita como instrucción transaccional (Transactional Strategies Instruction).

Además de las dos cuestiones formuladas por Maria (1987), consideramos necesario proceder al análisis de otras dos más y que están relacionadas con la concreción de los objetivos que se pretenden lograr y con la secuenciación de los contenidos seleccionados como relevantes. Por tanto, una tercera cuestión es la que hace referencia a ¿cuándo enseñar? La secuenciación de objetivos, contenidos y actividades constituye uno de los retos básicos que plantea el diseño de procesos de E-A y una de sus propiedades más relevantes. Para dar respuesta a esta cuestión, podemos tomar como referente la conceptualización de la comprensión como un proceso multinivel y que, posiblemente, tenga su origen en la distinción que propuso Bartlett (1932) entre representación superficial del texto y representación mental construida por el lector. Desde la década de los 80 , los estudios sobre la comprensión se han fundamentado en el supuesto de que, en el proceso de construcción del conocimiento, se pueden diferenciar distintos niveles de representación. El debate, generado inicialmente en torno a los 
contenidos de las representaciones mentales elaboradas, daría lugar, posteriormente, a una controversia más extensa acerca de la naturaleza y número de niveles que definen el proceso de construcción del conocimiento: nivel superficial, nivel semántico y modelo de situación (van Dijk y Kintsch, 1983); modelo mental (Johnson-Laird, 1983); estructuras lingüísticas, proposiciones y estructura de redes semánticas (Frederiksen y Donin, 1991); comunicación y género discursivo (Sovik, Samuelsten y Flen, 2000); representación proposicional, jerarquización de las ideas, modelo mental y expresión/comunicación (Escoriza, 2003), entre otros. Si analizamos la naturaleza de los diferentes niveles de representación que han sido propuestos, podremos establecer una secuencia en la ejecución de determinados procesos cognitivos, relacionados con la construcción de cada uno de dichos niveles. Así, por ejemplo, la actividad de aprendizaje del alumno/a se centraría inicialmente en el análisis de una de las propiedades del discurso escrito, la coherencia y, consecuentemente, tendría como objetivo seleccionar toda la información que el alumno/a considere necesaria en función de las expectativas personales formuladas, conocimientos previos, etc. y que se correspondería con el primero (o con los dos primeros) de los niveles propuestos: superficial, semántico (van Dijk y Kintsch, 1983); estructuras lingüísticas, proposiciones (Frederiksen y Donin, 1991); representación proposicional (Escoriza, 2003). A este nivel la finalidad general de la actividad de aprendizaje es la de tratar de comprender el sistema de significados formulado de manera explícita en el discurso escrito: ideas que comparten el mismo argumento y que definen la base semántica de su contenido (construcción de la coherencia interproposicional, microestructura, base textual, relación semántica interproposicional). Sanders y Noordman (2000) consideran que las relaciones de coherencia, entendidas como las relaciones significativas que conectan dos segmentos del texto, son una de sus propiedades básicas, ya que hacen posible la construcción de representaciones mentales igualmente coherentes. Comprender un discurso escrito supone, entre otros objetivos, construir de forma progresiva una representación coherente de su contenido. Algunas de las operaciones cognitivas susceptibles de ser ejecutadas a este nivel podrían ser las macrorreglas de supresión y generalización que proponen van Dijk y Kintsch (1983), las reglas para resumir mencionadas por Brown y Day (1983), el proceso cognitivo que sugieren King, Biggs y Lipsky (1984), las cuatro reglas aplicadas por Pressley y Woloshyn (1995), la composición operacional de las Acciones cognitivas de Subrayar y Resumir (Escoriza, 2003), etc.

Una vez que la información considerada como necesaria ha sido debidamente seleccionada, procede concretar su estructura interna, es decir, categorizar las ideas en función de su naturaleza inclusora: nivel de género discursivo (Sovik et al. 2000); jerarquización de las 
ideas (Escoriza 2003). La estructura de un discurso escrito es otra de sus propiedades fundamentales ya que, entre otras cosas, comprender implica construir una representación mental organizada. Para Sanders (1992), la estructura es una de las propiedades cruciales tanto del propio discurso escrito como de las representaciones cognitivas que los lectores/as han elaborado. La comprensión de la estructura del discurso escrito es considerada por Novak (2002) como uno de los factores esenciales en el proceso de construcción y reconstrucción de sistemas de significados por parte del alumno/a, hasta el punto de atribuir las causas de las dificultades, en la realización de aprendizajes significativos, a la elaboración de jerarquías proposicionales inadecuadas o, si se prefiere, a las dificultades en la elaboración de jerarquías proposicionales adecuadas. A este nivel, las operaciones cognitivas susceptibles de ser ejecutadas como pertinentes estarían relacionadas con la categorización de las ideas en información supraordinada e información subordinada y, por tanto, con dos de las macrorreglas (generalización y construcción) propuestas por van Dijk y Kintsch (1983) y con las operaciones cognitivas consideradas como componentes de las Acciones cognitivas Jerarquización de las ideas y Mapa conceptual (Escoriza, 2003).

Si los contenidos expresados en el discurso escrito que tratamos de comprender son coherentes y están debidamente estructurados, se habrá cumplido una de las condiciones básicas que propone Ausubel (1976) para que pueda generarse la construcción de representaciones mentales significativas. Otra de las condiciones es que el alumno/a posea los conocimientos previos necesarios. Una vez que han sido comprendidas debidamente tanto las relaciones de coherencia proposicional como las relaciones de subordinación existentes entre las ideas, es cuando en el proceso de comprensión adquiere una especial relevancia la interacción dinámica que debe producirse entre dos unidades: el modelo mental que activa el alumno/a (coherencia y estructura de representaciones mentales elaboradas) y el modelo científico (coherencia y estructura de los contenidos del discurso escrito). El objetivo de la actividad cognitiva del alumno/a será el de tratar de integrar, de forma sustantiva y no arbitraria, en su estructura cognitiva ya existente la información no conocida, produciéndose, como consecuencia, una reorganización o modificación del modelo mental activado inicialmente: modelo de situación (van Dijk y Kintsch, 1983), modelo mental (Johnson-Laird, 1983), estructura de redes semánticas (Frederiksen y Donin, 1991). La construcción de representaciones mentales, a este nivel, implica ejecutar una serie de operaciones cognitivas (especificación de la información conocida y no conocida, identificación de la información implícita, explicitación de los conflictos cognitivos, etc.), relacionadas con la interacción que debe darse entre modelo mental y 
modelo científico, con la finalidad de que la actividad de lectura se configure como un proceso funcional integrativo: la comprensión no como derivación del significado sino como un proceso de construcción del conocimiento (Escoriza, 1996b).

Si asumimos que la internalización de los instrumentos psicológicos, tal y como propone Karpov (1995), supone no sólo la comprensión de cierto conocimiento lingüístico sino también la capacidad para poderlo expresar o comunicar y aplicar, es evidente que deberemos seleccionar una serie de operaciones cognitivas relacionadas con la escritura considerada igualmente como un instrumento de aprendizaje (Escoriza, 1998b, 2003, en prensa; Escoriza y Boj, 1997). Escribir para aprender/escribir para expresar o comunicar lo aprendido, tienen la propiedad de hacer más explícito el modelo mental elaborado durante la actividad de lectura, ayuda a clarificar las comprensiones construidas, facilita la organización y reestructuración del conocimiento, etc.; en definitiva, la escritura como instrumento para compartir y para consolidar el conocimiento: nivel comunicativo (Graesser, Millis y Zwaan, 1997; Sovik et al. 2000); expresión/comunicación del conocimiento (Escoriza, 2003). Para concretar las operaciones cognitivas susceptibles de ser seleccionadas como pertinentes, podemos tener en cuenta una serie de aportaciones relacionadas con el análisis del proceso de escritura (Hayes y Flower, 1980; Bereiter y Scardamalia, 1987; Englert et al. 1991): generación de ideas, estructuración interna del contenido, construcción de la progresión temática, revisión.

Otra cuestión es la de ¿por qué enseñar? Existe un evidente consenso en asumir que la finalidad de la enseñanza de estrategias de comprensión es que el alumno/a aprenda a aprender. Este objetivo ha visto incrementado su valor educativo a medida que se han ido sucediendo los datos en apoyo de la incidencia que, en los contextos educativos escolares, habría de tener la propuesta formulada por Torgesen $(1977,1991)$ acerca de la explicación de las Dificultades de Aprendizaje: déficits en el conocimiento, selección y aplicación de estrategias. El llamado déficit estratégico es interpretado como una manifestación de que las personas con Dificultades de Aprendizaje pueden tener problemas a la hora de proceder a la planificación de secuencias de procesos cognitivos orientados a la realización de una actividad de aprendizaje, controlar su actividad cognitiva y modificarla si ello fuera necesario en función de si los objetivos se están logrando o no y en qué medida. Los defensores del modelo estratégico sostienen que la discrepancia entre CI y rendimiento escolar se debe a la incidencia del déficit estratégico. Dicha discrepancia es considerada como el criterio determinante que posibilita la identificación de las personas con dificultades de aprendizaje. Los resultados obtenidos en un 
estudio realizado con estudiantes universitarios (Escoriza, 2002) pusieron de manifiesto la generación de una serie de dificultades identificables a nivel operatorio o procedimental del conocimiento estratégico que suelen aplicar durante las actividades de aprendizaje: no explicitan las operaciones cognitivas adecuadas, no asignan, o asignan de forma incorrecta, objetivos específicos a las Acciones y a las operaciones cognitivas expuestas, el número de operaciones cognitivas mencionadas es claramente insuficiente, no establecen las necesarias relaciones entre cognición-motivación y formulan secuencias inadecuadas de objetivos, de acciones y de operaciones cognitivas.

Con independencia de la gravedad y/o persistencia del déficit estratégico evaluado en sujetos concretos, su incidencia se hace evidente en las dificultades que se generan en el empleo de la lectura y de la escritura como instrumentos de aprendizaje. Suele darse una discrepancia muy significativa entre el empleo del lenguaje oral y el empleo del lenguaje escrito en el aprendizaje de los contenidos escolares. Nos solemos encontrar con casos aparentemente paradójicos: el alumno/a no tiene dificultades para comprender conceptos cuando el instrumento mediador es el lenguaje oral, pero sí cuando el instrumento de aprendizaje ha de ser única y exclusivamente el lenguaje escrito. Esta necesidad educativa identificada requiere una respuesta adecuada orientada a promover el conocimiento estratégico mediante el diseño y desarrollo de actividades de aprendizaje mediatizado (Escoriza, 1996a, 1998a). De no ser así, se generará como una forma más de experiencia cotidiana que podría propiciar el desarrollo de un conocimiento estratégico espontáneo caracterizado por ser fragmentario, poco sistemático, organizado de forma inadecuada e inconsciente. La revisión efectuada por Gersten, Fuchs, Williams et al. (2001) acerca de la investigación realizada sobre la enseñanza de estrategias en alumnos con dificultades de aprendizaje les llevó a formular dos recomendacioness básicas con la finalidad de mejorar los procesos de comprensión: incrementar el empleo de procesos instruccionales mediados socialmente y la necesidad de enseñar conjuntos de estrategias. Leer para comprender/leer para aprender comprendiendo es una actividad cognitiva compleja que requiere una determinada competencia estratégica, tanto a nivel operatorio o procedimental como a nivel instrumental o funcional. La calidad del proceso de construcción del conocimiento depende, en gran parte, del conocimiento estratégico que el alumno/a puede activar y aplicar de manera efectiva y eficaz durante la actividad de lectura. La comprensión de un determinado discurso escrito requiere la ejecución deliberada y propositiva de un plan cognitivo que permita construir la coherencia proposicional, concretar su estructura interna, 
establecer relaciones sustantivas entre el modelo mental y el modelo científico y poder expresar/comunicar las comprensiones elaboradas.

La necesidad, cada vez más imperiosa, de dar una respuesta adecuada a todas estas cuestiones ha propiciado la generación de una intensa actividad investigadora orientada a lograr la identificación de las estrategias más relevantes y pertinentes cuya ejecución se supone que permitiría mejorar de forma significativa los procesos de aprendizaje del alumnado (Robinson, 1946; Tharp, 1982; Palincsar y Brown, 1984; Bereiter y Bird, 1985; Sánchez, 1989; Pressley, Johnson, Symons et al., 1989; Vidal-Abarca y Gilabert, 1991, 1994; Englert y Mariage, 1991; Beltrán, 1993, 1998; Carriedo y Alonso, 1994, 1995; Carrell, Gajdusek y Wise, 1998; Schraw, 1998; Pressley, 1998; King y Johnson, 1999; Sovik et al. 2000; Willoughby, Word, McDermott et al., 2000; Willoughby, Wood y Kraftcheck, 2003; Gersten et al. 2001; Smolkin y Donovan, 2001; De Corte, Verschaffel y Van de Ven, 2001; Bimmel, 2001; Elosúa, García Madruga, Gutiérrez et al., 2002; Schirmer, 2003; Escoriza, 2002, 2003, 2004ab; Guthrie, Wigfield, Barbosa et al. 2004; Braten y Samuelstuen, 2004; Connor, Morrison y Petrella, 2004; Kendeou y van den Broek, 2005, etc.).

En resumen, proceder al diseño de un proceso de E-A relacionado con la comprensión del LE requiere posicionarse a favor o en contra de las alternativas expuestas y que han ido surgiendo como respuestas posibles a las cuestiones planteadas:

A. ¿Por qué enseñar?: Con la finalidad de promover el desarrollo del conocimiento estratégico, tanto a nivel operatorio o procedimental cómo a nivel instrumental o funcional.

B. ¿Qué enseñar?:

a. Habilidades discretas, Estrategias individuales, listas o repertorios de habilidades o de Estrategias.

b. El proceso de comprensión como proceso holístico (de principio a fin), es decir, como una secuencia integrada de estrategias.

C. ¿Cuándo enseñar?:

a. Secuenciación de las Estrategias de acuerdo con la sucesión de fases en las que ha sido segmentado el proceso de comprensión: antes, durante o después de la actividad de lectura.

b. Secuenciación de las Estrategias en función de la ordenación de los objetivos específicos que han sido formulados: Selección, Organización, Elaboración/Integración y Expresión/Comunicación del conocimiento. 
D. ¿Cómo enseñar?:

a. Instrucción directa o Enseñanza explícita.

b. Enseñanza Recíproca.

c. Instrucción Transaccional.

\section{Selección y Secuenciación de Objetivos y Contenidos}

La selección y secuenciación de objetivos, acciones y operaciones cognitivas que vamos a analizar se fundamentan en:

a. Los datos aportados por un serie de investigaciones (ver introducción) realizadas con el propósito de concretar las Estrategias consideradas como las más adecuadas para promover la competencia estratégica del alumnado.

b. La teoría de la Actividad desarrollada por Leontiev (1990). De acuerdo con Leontiev (1990), la Actividad no es un concepto general, sino que es entendida como una unidad específica que responde a una necesidad (meta, motivo) determinada, generada en contextos culturales y en condiciones también específicas. El motivo/la meta es la característica principal que permite diferenciar a una actividad concreta de otras y condiciona, por tanto, su estructura interna, orientación, finalidad, etc. El motivo, en definitiva, es el que le da sentido y define la naturaleza de una actividad específica. En nuestro caso, la actividad específica es la lectura/escritura y cuya meta/motivo es que el alumno/a aprenda de forma significativa (el aprendizaje como construcción del conocimiento). En el análisis de la estructura interna de la actividad, los componentes básicos son las Acciones, relacionadas con objetivos específicos de naturaleza instrumental con respecto a la meta. Son diferentes de la meta, pero dependientes de ella. En consecuencia, la realización de cualquier actividad implica el logro de una serie de objetivos concretos mediante la ejecución de una secuencia integrada de Acciones. Toda acción tiene dos aspectos, el intencional (el objetivo particular: lo que debo lograr) y el operacional (la composición operacional: cómo puedo lograrlo). Una vez que ha sido concretado el objetivo de cada una de las acciones procede determinar el proceso relativo al aspecto operacional, es decir, qué operaciones debemos ejecutar o aplicar con la finalidad de lograr alcanzar el objetivo asignado a cada una de las acciones y en la secuencia establecida. La interpretación de la Actividad de lectura/escritura, como una forma de actividad específica (Escoriza, 2003), que responde a necesidades (meta/motivo) generadas en contextos socioculturales concretos (comprensión de los contenidos escolares) nos permite considerar a dicha actividad, 
como un sistema funcional con su propia estructura interna y cuyos componentes interactúan a diversos niveles: Actividad, Acción y Operación.

Teniendo en cuenta lo expuesto con anterioridad, proponemos la siguiente selección de objetivos instrumentales específicos (¿por qué enseñar?) y contenidos (¿qué enseñar?), así como su secuenciación correspondiente (¿cuándo enseñar?). En cuanto a la especificación de las características de las acciones educativas más adecuadas (¿cómo enseñar?), nos remitimos a los principios formulados desde la perspectiva sociocultural por su relevancia y pertinencia con respecto a la explicación de los procesos de E-A que se desarrollan en las aulas (Rosenshine y Meister, 1994; Escoriza, 1996a, 1998; Greenway, 2002; Lensky y Nierstheimer, 2002).

\section{Aplicación de las operaciones cognitivas cuya ejecución tiene como objetivo Seleccionar la} Información relevante

Ya hemos indicado anteriormente que la realización de cualquier actividad específica implica el logro de una serie de objetivos concretos mediante la ejecución de una secuencia integrada de Acciones. En consecuencia, la asignación de objetivos instrumentales a cada una de las Acciones nos permite concretar la organización de dicha secuencia y definir el sistema cuyo funcionamiento va a posibilitar alcanzar la meta prevista. El primer objetivo es el de Seleccionar la información que consideremos relevante en función de los conocimientos previos y de la meta establecida. Las acciones cognitivas relacionadas con este objetivo son Subrayar y Resumir, compuestas por una serie de operaciones cognitivas cuya aplicación práctica va a permitir alcanzar el logro de dicho objetivo.

Tabla 1. Aspectos de las Acciones Cognitivas Subrayar y Resumir

\begin{tabular}{|l}
\hline $\begin{array}{l}\text { ACCIONES COGNITIVAS: } \\
* \text { Subrayar } \\
* \text { Resumir }\end{array}$ \\
\hline Objetivo: Seleccionar Información \\
\hline Composición Operacional: \\
a. Construcción del argumento compartido \\
b. Diferenciar entre información Nueva e información Dada \\
c. Suprimir la información trivial o Seleccionar la información \\
relevante \\
d. Identificación de los marcadores discursivos, referentes, etc. \\
e. Sustituir secuencias de ideas por otra más inclusora
\end{tabular}


El proceso de intervención educativa lo iniciamos con la negociación conjunta del concepto de progresión temática (Escoriza, 2003), considerada como una estructura discursiva empleada para representar la relación semántica existente entre información nueva e información dada o redundante (es, igualmente, un concepto de gran utilidad para la enseñanza de la composición escrita). La aportación sucesiva de información nueva es la que va a hacer posible que el tema se vaya desarrollando debido a la relación de continuidad semántica que se va produciendo entre las ideas que comparten el mismo argumento. Pero también nos encontramos con otro tipo de información, cuya finalidad es la de mejorar la claridad expositiva, facilitar la comprensión de la información nueva, etc. La información dada o redundante tiene la propiedad de expresar el mismo significado que la información nueva, pero empleando para ello unidades lingüísticas diferentes, es decir, las mismas ideas pero expresadas de forma distinta. El nivel de redundancia variará de un discurso a otro dependiendo de la cantidad de frases que repiten la esencia de las ideas expresadas mediante la información nueva. Ahora bien, puede darse el caso de que parte de la información nueva o de la información dada no guarde ningún tipo de relación semántica con la mayoría de los enunciados del discurso escrito o que algunas ideas sean expresadas de forma ambigua o confusa. La cuestión que se plantea es: de toda la información que se expone en un discurso escrito, ¿qué ideas, enunciados, información, debemos seleccionar? o ¿qué criterios deberíamos aplicar para seleccionar la información importante y prescindir de la que no lo es? Es esta una de las inquietudes básicas que manifiestan los alumnos/as ante la preparación de las pruebas de evaluación. La respuesta la tenemos en el concepto expuesto de progresión temática. Un discurso escrito tiene continuidad temática si la información aportada además de ser nueva es coherente. Estos son, por tanto, los dos criterios que podemos aplicar para seleccionar la información y para determinar si es importante o no. Además de la información que siendo nueva es también coherente, es aconsejable seleccionar toda aquella información redundante que consideremos como necesaria para comprender a la información nueva seleccionada. Deberemos prescindir, por tanto, de la información categorizada como contradictoria (incoherente), ambigua, confusa o redundante, ya que no suponen una ayuda para interpretar a otras ideas. Para lograr el objetivo, consistente en Seleccionar la información relevante, podemos ejecutar las siguientes operaciones cognitivas:

a. Construcción del argumento compartido. La ejecución de esta operación cognitiva nos va a permitir concretar el grado de coherencia existente entre los diferentes enunciados de un discurso escrito. Según Sanders (1992), uno de los aspectos de un discurso escrito es la coherencia referencial: un discurso escrito es coherente si existe una referencia repetida del 
mismo tema, vía solapamiento argumental, es decir, un conjunto de ideas son coherentes si comparten el mismo referente (tema, título, idea principal). Un ejemplo de aplicación de esta operación cognitiva en los discursos escritos DE-1 y DE-2 (ver Anexo) es el siguiente. La totalidad de las ideas expresadas en el DE-1 comparte el referente animales y útiles. El discurso escrito tiene continuidad temática porque las ideas nuevas van aportando información que siempre tiene como referente compartido el tema de las utilidades de los animales. En el DE-2, la relación semántica global existente entre la mayoría de las ideas la podemos identificar en el referente compartido por todas ellas: la Balanza de Pagos, estructura o composición. Existe una referencia repetida del mismo tema, ya que la mayor parte de los enunciados tienen como referente a la estructura de la Balanza de Pagos.

b. Diferenciación entre información nueva e información dada. Cuando el referente compartido ha sido identificado, procede seleccionar la información nueva mediante la cual se va desarrollando y que, por tanto, responde al criterio de coherencia referencial. Así, por ejemplo, en el DE-1, procede seleccionar, entre otras, las siguientes ideas: "los animales son útiles por muchas razones. Los animales nos dan comida...Los animales son útiles para el transporte...etc.". Toda esta información es relevante ya que en ella concurren los dos criterios ya indicados: es nueva y es coherente. Ahora bien, ¿sería conveniente seleccionar también las ideas del tipo siguiente?: "Por ejemplo, conseguimos carne de las vacas y de los cerdos...". Esta información no es nueva, ya que repite la esencia de la idea ya expresada anteriormente, los animales nos dan comida, y además disponemos de un marcador discursivo ("por ejemplo") que nos informa de que la frase que viene a continuación pretende clarificar a la idea que ya ha sido expuesta. Es, por tanto, información dada o redundante (en este tipo de información sólo se da uno de los dos criterios expuestos: la coherencia referencial). Su selección ya no dependerá de su propia naturaleza, sino de los conocimientos previos del lector/a. En el caso del DE-2, la ejecución de esta operación cognitiva puede ser más problemática. Nos encontramos con una serie de ideas que podemos categorizar como información nueva, pero ¿concurre en ellas además el criterio de coherencia referencial? Por ejemplo: "Las transacciones económicas llevadas a cabo requieren la existencia de alguna forma de pago internacional". "El valor de las importaciones y de las exportaciones, de bienes materiales, queda registrado en la balanza de mercancías...”. ¿Es aconsejable proceder a la selección de las dos?, ¿las dos son información nueva y además coherente?, ¿Forman parte de la información redundante? Para poder dar una respuesta adecuada a estas cuestiones, es necesario tener en cuenta el resultado obtenido en la ejecución de la operación cognitiva anterior. El referente, el tema, el argumento, concretado fue: composición de la Balanza de Pagos. ¿Este dato permite 
tomar decisiones con mayor facilidad? En este punto es aconsejable poner de manifiesto la importancia que tiene la ejecución adecuada de la operación cognitiva consistente en la construcción del argumento compartido. El lector/a podrá comprobar que el proceso de toma de deciones será diferente en función del referente compartido que haya sido elaborado. En un estudio realizado con alumnos/as universitarios las discrepancias fueron evidentes: los intercambios económicos de un país; factores influyentes en la economía española; cómo fluctúa la economía; repercusiones de la economía global en la economía local; la balanza de pagos, etc. Fácilmente podemos comprobar que el tipo de información susceptible de ser seleccionada como relevante depende del referente concretado, ya que el criterio de coherencia referencial se podrá aplicar a unas u otras ideas en función de si están o no relacionadas semánticamente.

c. Supresión de la información trivial (irrelevante, ambigua, confusa, contradictoria, redundante no necesaria). Esta operación cognitiva se corresponde con la macrorregla de supresión que proponen van Dijk y Kintsch (1983): dada una secuencia de proposiciones, suprimir todas aquellas que no se configuran como una condición semántica para la interpretación de la siguiente. Ha sido empleada como referente, en diversos estudios, para la concreción de las operaciones cognitivas relacionadas con la acción de Resumir (Brown y Day, 1984; King et al. 1984; Winograd, 1985; Pressley y Woloshyn, 1995; Thiede y Anderson, 2003). En el DE-1, podemos no seleccionar o suprimir, sin que ello afecte a la comprensión de otras ideas, las frases de "Cabalgar es muy divertido. Pescar es un deporte...", ya que es una información redundante que no es necesaria para entender la información nueva con la que está relacionada semánticamente "los animales nos proporcionan diversión". En el DE-2, la frase "las zonas rurales pueden quedar desiertas, debido al incremento emigratorio hacia las grandes ciudades" no guarda tampoco ninguna coherencia relacional con la balanza de servicios y su supresión no influye en la comprensión de dicho concepto.

d. Identificación funcional de los marcadores discursivos, referentes, conectores. Todos estos elementos lingüísticos tienen en común que son indicadores de las relaciones de coherencia existentes entre dos o más enunciados del discurso escrito. En el DE-1, encontramos dos tipos de marcadores que hacen más explícita las relaciones de coherencia: "Los animales nos dan comida. Por ejemplo..."; “Los animales también...”. En el DE-2, los podemos identificar en: "Si un país compra a otro naranjas...Sin embargo..."; "Los estados también..."; "Pero la emigración también...". Veamos el siguiente enunciado: "Se ha encontrado un tipo de dinosaurio cuya evolución no ha sido a partir de los animales marinos, sino de los animales terrestres, descendientes, a su vez, de los peces". Interpretación realizada por dos alum- 
nos: "Este texto trata del descubrimiento de una especie de dinosaurio evolucionado a partir de los peces (como todos ellos)"; "Los peces evolucionaron y poco a poco pudieron ir por tierra: anfibios, reptiles. Algunos se hicieron terrestres y de estos aparecieron los dinosaurios, es decir, que evolucionaron de los animales terrestres". La comprensión correcta de este enunciado requiere comprender la continuidad referencial de la que nos informan los distintos marcadores que se van incluyendo: "cuya evolución no ha sido... sino... descendientes, a su vez...".

e. Sustitución de una secuencia de proposiciones por otra más inclusora. Es una operación cognitiva propia de la Acción de Resumir y no de Subrayar. Es otra de las macrorreglas propuestas por van Dijk y Kintsch (1983) y que permite reducir la información expresada mediante la categorización de una secuencia de ideas en función de su naturaleza inclusora. Supongamos un discurso escrito en el que se exponen los conceptos de leche, mantequilla y yogur. Esta secuencia de conceptos puede ser sustituida por otro más inclusor: productos lácteos. En el DE-1 podemos sustituir la totalidad del contenido por la frase: "Los animales son útiles porque nos proporcionan comida y diversión, nos ayudan en el trabajo y el transporte, $y$, obtenemos además diversos productos". En el DE-2, la secuencia de frases que va desde "Los ciudadanos de diversos países... hasta... hacer inversiones, etc." la podemos sustituir por otra más inclusora "Los pagos internacionales se realizan mediante divisas".

2. Aplicación de las operaciones cognitivas cuya ejecución tiene como objetivo Organizar la Información en función de su naturaleza inclusora

La ejecución de las operaciones cognitivas que definen la composición de las Acciones cognitivas Jerarquización de las ideas y elaboración de un Mapa Conceptual, y cuya finalidad es la de organizar en categorías informativas a las ideas seleccionadas, se fundamenta en la relevancia que en el proceso de comprensión tiene el conocimiento de la estructura del discurso escrito. Conocer dicha estructura implica conocer las distintas formas potenciales con las que el autor/a puede organizar la progresión temática. Todos los discursos escritos están estructurados de una forma lógica y sistemática y, por tanto, su reconstrucción por parte del lector/a constituye una competencia importante en el proceso de comprensión del LE, debido a su incidencia en los procesos de construcción de representaciones mentales y de recuerdo-recuperación de los sistemas de significados construidos. 
Tabla 2. Aspectos de las Acciones Cognitivas Jerarquizar las ideas y Mapa Conceptual

\begin{tabular}{|l|}
\hline $\begin{array}{l}\text { ACCIONES COGNITIVAS: } \\
\text { * Jerarquización de las Ideas } \\
\text { * Mapa Conceptual }\end{array}$ \\
\hline Objetivo: Organización Interna del Contenido \\
\hline Composición Operacional: \\
a. Especificación del género discursivo \\
b. Concreción del título \\
c. Identificación o elaboración de la Idea Principal \\
d. Identificación o elaboración de las Ideas Subordinadas \\
e. Especificación de los niveles de elaboración \\
f. Análisis de las claves lingüísticas estructurales \\
g. Representación gráfica de las relaciones de subordinación \\
\hline
\end{tabular}

a. Especificación del género discursivo. Conocer el género discursivo al que pertenece el discurso escrito que pretendemos comprender facilita la actividad de aprendizaje, ya que constituye una guía muy útil para la categorización de su contenido. Dicho conocimiento ayuda a organizar las ideas, durante el proceso de comprensión, y posibilita su integración en una representación mental estructurada. En general, los discursos escritos (narrativos, descriptivos, expositivos, argumentativos) se diferencian entre sí por las características de los componentes siguientes: finalidad, estructura interna, contenido y marcadores discursivos (los DE-1 y 2, son discursos expositivos).

b. Concreción del título. La ejecución de esta operación cognitiva tiene como antecente directo a la denominada construcción del argumento compartido (componente de las Acciones de Subrayar y Resumir), ya que es la que nos va a permitir construir una jerarquía adecuada de ideas en base al criterio del número de ideas que lo comparten, o, en otros términos, el número de ideas que tienen como referente a otra idea más supraordinada. Una vez que el argumento compartido ha sido elaborado, podemos concretar la información más supraordinada que se va a configurar como referente y la que, en consecuencia, va a permitir determinar la relación de subordinación existente entre las ideas en las que concurre el criterio de coherencia referencial. Es aconsejable, incluso en los discursos escritos que ya tienen título, comprobar su funcionalidad como referente. El título más adecuado es siempre aquel que nos permite comprender con mayor facilidad la relación semántica existente entre las ideas que lo 
desarrollan. Por ejemplo, de los títulos siguientes, concretar el que pueda ser el más adecuado para comprender el DE-1 (los animales y los humanos; la granja de animales; los animales nos dan la vida; utilidades de los animales) o el DE-2 (las relaciones económicas internacionales; estructura o composición de la balanza de pagos; factores que influyen en la economía española). En las pruebas de evaluación hemos podido comprobar (Escoriza, 2004a) que los títulos y subtítulos carecen de valor referencial o no se les otorga el valor adecuado a este tipo de marcadores discursivos. Tanto los títulos como los subtítulos (Escoriza, 2003) cumplen una doble función: nos proporcionan información acerca de la estructura global del discurso escrito y actúan como referentes que nos anuncian el tipo de información que va ser expresada en la progresión temática. Las consecuencias no deseadas, derivadas de su infravaloración, son de naturaleza muy diversa, pero todas ellas comparten una particularidad: el alumno/a tiene dificultades para relacionar el título con las ideas que lo desarrollan y, por tanto, las preguntas con las respuestas. Estas dificultades se ponen de manifiesto cuando los alumnos/as formulan cuestiones del tipo “¿esta pregunta a qué tema pertenece?, ¿a esta pregunta está bien contestar de esta manera?, ¿cómo empieza la respuesta a esta pregunta? o en aquellos casos en los que las relaciones son tan arbitrarias que generan situaciones del tipo siguiente: un alumno que cuando tenía que responder a una pregunta formulaba siempre la misma petición ¿cómo empieza en el libro? O el caso de otra alumna que, cuando se le entregaba el examen con las preguntas, no las tenía en cuenta y expresaba todo lo que había estudiado del tema. Al final del examen solía poner una nota que decía: profesora califique lo que Vd. crea que he puesto bien.

c. Identificación o elaboración de la Idea Principal. La ejecución de esta operación cognitiva nos va a permitir identificar el contenido más general o global del discurso escrito, ya que se configura como la idea cuyo significado puede ser relacionado con la totalidad de las ideas que hemos seleccionado porque en ellas concurre el criterio de coherencia referencial (información nueva que además es coherente e información dada que la desarrolla). Forma parte de la información supraordinada, ya que expresa el mayor nivel de inclusión al ser el referente compartido por todas las ideas que desarrollan al tema. La aplicación de la macrorregla de construccción (van Dijk y Kintsch, 1983) permite la elaboración de una idea más inclusora que sustituya a una serie de ideas menos inclusoras con las que comparte el mismo argumento y de las que es su referente. En el DE-1, la idea que responde a estas características es: “los animales son útiles por cuatro razones: nos dan comida, etc...”. En el DE-2 es: “La Balanza de Pagos se compone de cuatro subbalanzas: mercancías, servicios, transferencias y por cuenta de capital". 
d. Identificación o elaboración de las Ideas Subordinadas. Son todas aquellas ideas que tienen como referente directo o inmediato a la Idea Principal y que pueden ser relacionadas semánticamente con un determinado conjunto de ideas menos inclusoras. Se configuran, por tanto, como categorías informativas a las que pertenecen un conjunto de ideas de las que la idea subordinada es el referente directo. En el DE-1, la idea "nos dan comida" es una de las ideas subordinadas ya que, por una parte, tiene como referente directo a la Idea Principal (IP) (dar comida es una de las utilidades) y, por otra, es una categoría informativa a la que pertenecen un conjunto de ideas relacionadas semánticamente entre sí (coherencia relacional) pero no con otras ideas, como por ejemplo "cabalgar es divertido". En el DE-2, la idea "la balanza de mercancías registra el valor de las importaciones y exportaciones..." es igualmente una idea subordinada ya que en ella concurren las mismas características que ya hemos indicado anteriormente.

e. Especificación de los diferentes niveles de elaboración de las ideas subordinadas. La ejecución de esta operación cognitiva tiene como finalidad determinar el nivel de diferenciación conceptual progresiva o grado de inclusividad al que ha llegado el autor/a de la composición escrita en cada una de las ideas subordinadas. En el DE-1, el desarrollo conceptual solamente lo podemos considerar como de nivel uno y para la totalidad de las ideas subordinadas (Idea subordinada: nos dan comida; diferenciación progresiva: carne de las vacas).

f. Análisis de las claves lingüísticas estructurales. Son unidades lingüísticas que proporcionan información acerca de las relaciones jerárquicas existentes entre las ideas. Según sostiene Sanders (1992), la estructura relacional del discurso escrito está marcada lingüísticamente mediante el empleo de determinadas palabras, frases, etc. (primero, segundo...; lo más importante es...; quiero destacar o conviene resaltar...etc.).

g. Representación gráfica de la estructura jerárquica elaborada. Consistiría en la elaboración de un Mapa Conceptual en el que las ideas seleccionadas son representadas gráficamente en una secuencia estructurada que va de los conceptos más inclusores a los menos inclusores.

3. Aplicación de las operaciones cognitivas cuya ejecución tiene como objetivo la Elaboración/Integración del conocimiento

Una vez que se ha producido la comprensión tanto de las relaciones de coherencia como de las relaciones de subordinación existentes entre las ideas expresadas en el discurso escrito, es cuando estimamos oportuno proceder a la aplicación práctica de las operaciones 
cognitivas, que definen la composición de la Acción cognitiva Relacionar los conocimientos previos con los contenidos del discurso escrito, con la finalidad de poder lograr el objetivo instrumental específico que le ha sido asignado: la Elaboración/Integración del conocimiento. Desde el constructivismo y la teoría del aprendizaje significativo (Escoriza, 1998a; Kintsch, 1988), se viene destacando la relevancia e incidencia de los conocimientos previos en los procesos de internalización del conocimiento: lo que un alumno/a sabe determina lo que puede aprender de forma significativa. La consecución de este objetivo solamente podrá hacerse efectiva si se produce, por tanto, una relación sustantiva entre lo que el alumno/a ya sabe y lo que se considera que debe aprender. En consecuencia, han sido seleccionadas como pertinentes todas aquellas operaciones cognitivas relacionadas con: activación y aplicación de los conocimientos previos, la realización de procesos inferenciales y la integración de las ideas, de una forma coherente y estructurada, en la estructura cognitiva del alumno/a.

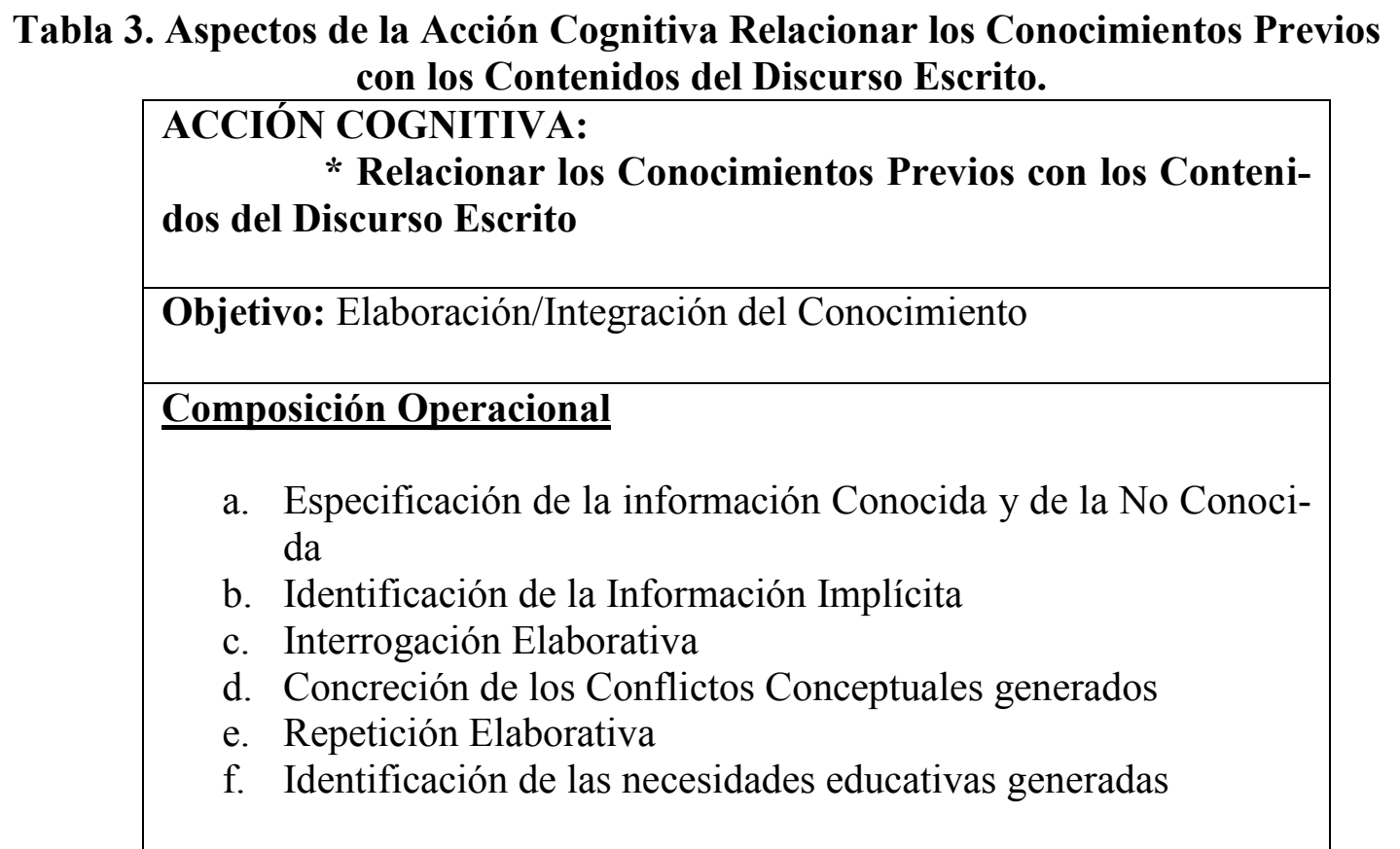

a. Especificación de la información conocida y de la no conocida. La explicitación de los contenidos ya conocidos y de los no conocidos adquiere una doble dimensión. Por una parte, hacen posible que el proceso de comprensión se configure como un proceso constructivo relacionando de forma sustantiva las ideas conocidas con las ideas no conocidas. Por otra, permite ser consciente de las modificaciones potenciales que se pueden producir en la estructura cognitiva del alumno/a. La ejecución de esta operación cognitiva tiene como finalidad categorizar, en conocida o en no conocida, la información seleccionada como relevante me- 
diante el Subrayado y/o el Resumen. La inclusión correcta de las ideas en una u otra categoría requiere la aplicación de unos criterios claros sobre cuándo el alumno/a considera que ha aprendido una serie de contenidos determinados. En consecuencia, proponemos que una información concreta sea categorizada como conocida cuando concurran las dos características siguientes: comprensión conceptual y capacidad para poderla expresar, comunicar o exponer. Para algunos alumnos/as una información es conocida cuando responde a alguno de los siguientes criterios (Escoriza, 2004a): le suena, le es familiar, si la comprende cuando la lee, etc. Si, por ejemplo, hemos seleccionado como relevante, en el DE-2, la siguiente información "La contabilidad, de las operaciones económicas con el exterior, se hace efectiva en la llamada Balanza de Pagos...", ahora lo que procede es determinar si el significado que en ella se expresa forma ya parte de nuestra estructura cognitiva, es decir, si la hemos comprendido y la podemos expresar. En caso contrario, deberá ser categorizada como no conocida.

b. Identificación de la información implícita. Una vez que la información no conocida ha sido debidamente concretada, es aconsejable tratar de identificar la información que ha omitido el autor/a pero que es considerada como necesaria para la construcción de las relaciones de coherencia y de subordinación existentes entre las ideas en base a la relevancia que adquieren las inferencias en el proceso de comprensión (Hansen, 1981; Graesser, Millis y Zwaan, 1997). En el DE-2, nos encontramos con conceptos que no han sido objeto de definición alguna: divisas, sistema monetario, inversiones a largo plazo, sector primario, mercancias, transferencias, etc. En este caso, lo procedente es concretar si la totalidad de los conceptos expuestos o solamente algunos de ellos constituyen información necesaria para comprender a la información no conocida y si dicha información las podemos aportar o no activando nuestros conocimientos previos. En el supuesto de que ello no sea posible, deberemos recurrir a la consulta de otras fuentes documentales o solicitar la correspondiente ayuda educativa (compañeros/as, profesorado).

c. Interrogación Elaborativa. Consiste en la autoformulación de preguntas durante la actividad de comprensión. Para Jansen (2002), la importancia de esta operación cognitiva se fundamenta en que juega un papel destacado en el aprendizaje autorregulatorio e independiente, ayuda de forma natural a la comprensión e incrementa el procesamiento activo del discurso escrito. Dependiendo del tipo de preguntas que un alumno/a se formule, la interrogación elaborativa tendrá una eficacia diferente, pero, en general, es de gran utilidad para controlar el proceso de comprensión, detectar necesidades educativas, explicitar conflictos cognitivos, clarificar conceptos, relacionar ideas, elaborar conclusiones, siendo además un procedimiento muy práctico con respecto a la autoevaluación. Si nos formulamos la pregunta ¿por qué no se 
registran en la subbalanza por cuenta de capital, las inversiones a corto plazo al igual que se hace con las de a largo plazo?, al tratar de darle una respuesta adecuada es seguro que todos los conceptos implicados quedarán integrados en nuestra estructura cognitiva. O esta otra: los procesos inflacionistas ¿afectan por igual a la relación ingresos/pagos en los registros de todas las subbalanzas?

d. Formulación explícita de los conflictos conceptuales generados. Un conflicto conceptual se genera cuando tomamos conciencia de la existencia de una clara discrepancia entre lo que sabemos y la nueva información. Un niño/a sabe que cuando lanza un objeto al aire inevitablemente cae al suelo. Un día observa en el aeropuerto que los aviones vuelan y no se caen. Pero no basta con que dicha experiencia, susceptible de generar una disonancia cognitiva, se haya producido. Es necesario, además, que el propio sujeto u otra persona más experta la hagan explícita. Ali (1991) considera necesario que el conflicto cognitivo sea creado o hecho consciente en base a la idea de que los seres humanos tienen una necesidad innata de resolver esta disonancia. Dicha autora sugiere que sea empleado como estrategia instruccional ya que, según ha podido constatar, genera mejoras notables en los resultados del aprendizaje. En el DE-1, encontramos la siguientes información"...productos que se emplean en medicina, proceden de los animales". Supongamos que el autor/a pone como ejemplo de este tipo de productos el veneno obtenido de las serpientes. Esta información puede generar una discrepancia entre el concepto de veneno como un producto que puede causar la muerte y su contrario, como un producto que puede ser empleado con la finalidad de impedirla. Si el conflicto lo hacemos explícito y el alumno/a siente la necesidad de resolverlo se habrá generado un aprendizaje comprensivo. Según Vosniadou (1991), ello se produce cuando el alumno/a logra integrar el modelo científico en su estructura cognitiva. La internalización del conocimiento implica hacer efectivo un proceso de reconciliación integradora, es decir, supone integrar en la estructura cognitiva los conceptos del modelo científico que, inicialmente, estaban en contradicción (discrepaban) con los conocimientos previos del alumno/a.

e. Repetición Elaborativa. El proceso de comprensión no se rige por la ley del todo o nada, sino que, por el contario, es un proceso de elaboraciones sucesivas. Es aconsejable leer varias veces un tema o unos contenidos con la finalidad de clarificar ideas, corregir errores conceptuales, etc. Si relacionamos la ejecución de esta operación cognitiva con las que hemos expuesto tanto en este apartado como en los anteriores, su finalidad está más que justitificada: comprobar el resultado obtenido en la ejecución de todas o algunas de las que consideremos como más funcionales según la naturaleza de los contenidos que intentamos comprender. 
f. Concreción de la ayuda educativa necesaria. Durante el proceso de comprensión pueden surgir dificultades cuya superación requiere el concurso o la ayuda de otras personas (compañeros/as, profesorado). La comprensión de un discurso escrito es una actividad durante la cual suelen darse dos tipos de aprendizaje: directo y mediatizado. Evidentemente, una parte de la información expresada, es posible que el alumno/a la pueda comprender por sí mismo (Zona de Desarrollo Real). Pero también puede ocurrir que otra parte, no menos importante, requiera la colaboración de una persona más experta, es decir, el proceso de comprensión como una forma de actividad de aprendizaje mediatizado (Zona de Desarrollo Próximo). Estas necesidades educativas pueden generarse en la ejecución de cualquiera (o en todas) de las siguientes operaciones cognitivas: información no conocida, información implícita, interrogación elaborativa y/o conflictos cognitivos.

4. Aplicación de las operaciones cognitivas cuya ejecución tiene como objetivo la Expresión/Comunicación del Conocimiento

Lo expuesto en los apartados anteriores ha tenido como denominador común el análisis de una serie de operaciones cognitivas relacionadas con el empleo de la lectura como instrumento de aprendizaje: leer para aprender. Se supone que si el alumno/a ha logrado seleccionar, organizar e integrar la información en su estructura cognitiva, mediante la ejecución de la secuencia de operaciones cognitivas expuestas, se habrá cumplido una de las condiciones indicadas como requisito para que una información determinada sea considerada como conocida: comprensión conceptual. La segunda condición es que el alumno/a la pueda expresar/comunicar/exponer/aplicar. Estas son, por tanto, las dos propiedades relevantes de lo que significa o implica el proceso de aprendizaje como proceso constructivo del conocimiento. La formulación del objetivo, consistente en promover en el alumnado la competencia para expresar el conocimiento, implica considerar a la escritura como un instrumento de aprendizaje (Escoriza, 2003, 2004ab). De esta manera leer para aprender y escribir para aprender se convierten en procesos transaccionales.

Tabla 4. Aspectos de la Acción Cognitiva Producir una Composición Escrita. ACCIÓN COGNITIVA:

* Producir una Composición Escrita

Objetivo: Expresión/Comunicación del Conocimiento

Composición Operacional: 

a. Generación de ideas
b. Jerarquización de las ideas
c. Secuenciación de las ideas
d. Construcción de la Progresión Temática
e. Revisión

a. Generación de las ideas. La ejecución de esta operación cognitiva requiere iniciar el proceso interactivo que debe producirse entre la lectura y la escritura como instrumentos de aprendizaje. Las ideas, que más tarde van a formar parte de la progresión temática, deberán ser las que ya hemos seleccionado como relevantes durante la actividad de lectura. Si los conocimientos que deseamos expresar son los correspondientes al DE-2, las ideas susceptibles de ser generadas serán todas aquellas que tienen como referente compartido a la temática relacionada con la composición de la Balanza de Pagos.

b. Jerarquización de las ideas. Una vez que ha sido concretada la información esencial que deseamos expresar, procede especificar las categorías en las que vamos a incluir todas aquellas ideas en las que concurren los criterios de coherencia referencial y relacional (las actividades de lectura y escritura como procesos transaccionales en la estructuración interna del contenido). Supongamos que hemos generado las siguientes ideas: los estados intercambian materias primas y realizan inversiones; los habitantes de un estado se van de vacaciones a otros países; los emigrantes envían dinero a sus países de origen; Alemania compra naranjas a España; España compra petróleo a Irán y gas natural a Túnez; la subbalanza de mercancías; la subbalanza por cuenta de capital, etc. De las ideas expresadas, las dos más inclusoras son: La subbalanza de mercancías y la subbalanza por cuenta de capital. Seguidamente, procede incluir, en dichas categorías, las ideas en las que concurren la propiedad de coherencia referencial: "Subbalanza de mercancías: los estados intercambian materias primas; Alemania compra naranjas a España; España compra petróleo a Irán y gas natural a Túnez". "Subbalanza por cuenta de capital: los estados realizan inversiones; los habitantes de un estado compran viviendas en otros países". Observamos que hemos escrito una idea que no guarda coherencia referencial con ninguna de las dos categorías anteriores: los emigrantes envían dinero a sus países de origen. Para solucionar la cuestión, tenemos dos opciones. Una es la de elaborar una categoría más y esta sería: subbalanza de transferencias. Otra, consistiría en suprimirla, en el supuesto de que no deseemos hacer referencia a este tipo de información en la composición escrita que pretendemos producir. 
c. Secuenciación de las categorías establecidas. Cuando las ideas han sido categorizadas en función de su naturaleza inclusora, procederemos a concretar su secuenciación en la progresión temática: por dónde empezar, cómo seguir y cómo acabar.

d. Construcción de la progresión temática. La ejecución de esta operación cognitiva tiene como finalidad producir el discurso escrito deseado: secuenciación de las ideas en la forma acordada, expresión del conocimiento con claridad, suficiencia, coherencia, objetividad, etc. (Escoriza, 2003, págs. 101-105).

e. Revisión. La finalidad principal que debe perseguir la ejecución de esta operación cognitiva es la de tratar de mejorar la calidad de la composición escrita producida. Implica la detección y corrección de los errores generados en la totalidad del proceso que se ha seguido para expresar el conocimiento elaborado durante la actividad de lectura e internalizado o integrado en la estructura cognitiva ya existente.

\section{Evaluación de las dificultades generadas en el proceso de comprensión del lenguaje es- crito}

El diseño y desarrollo efectivo y eficaz de un proceso de E-A requiere, entre otras cosas, que sean conocidas las dificultades de aprendizaje (DA) potenciales que se puedan generar. Dicho conocimiento puede posibilitar el hecho de que la intervención educativa sea de naturaleza preventiva y, si a pesar de ello se producen, que adquiera una finalidad correctora. En cualquier caso, solamente se podrá proporcionar la ayuda educativa necesaria y contingente si son conocidas debidamente las causas de las necesidades educativas identificadas. ¿Cómo podemos ayudar a un alumno/a a mejorar la calidad de sus Resúmenes, si no conocemos las causas de las dificultades que ha tenido en su realización?, ¿Cómo puede un alumno/a mejorar sus procesos de autoaprendizaje si desconoce las causas de las dificultades que tiene para comprender? La evaluación de las dificultades que se pueden producir en el proceso de comprensión del LE es aconsejable efectuarla en los dos componentes del conocimiento estratégico: el operatorio o procedimental y el instrumental o funcional (Escoriza, 2003). El primero hace referencia al conocimiento explícito de las Estrategias que el alumno/a aplica de forma sistemática durante la actividad de lectura. Forma parte, por tanto, del conocimiento procedimental y su evaluación nos debe proporcionar información acerca de los objetivos que pretende lograr mediante su ejecución y las acciones y operaciones cognitivas que ejecuta para lograrlos. Un estudio realizado con estudiantes universitarios nos permitió establecer una serie de categorías específicas de dificultades en la explicitación del conocimiento estratégico 
que han internalizado a lo largo de sus experiencias de literidad (los tipo de tareas, el procedimiento de evaluación y las categorias de dificultades establecidas, pueden verse en Escoriza, 2002, 2003). Mediante la evaluación del segundo componente lo que se pretende es obtener información acerca de los resultados derivados de la aplicación práctica de las diferentes operaciones cognitivas ejecutadas con la finalidad de Seleccionar, Organizar, Elaborar y Expresar el conocimiento (Tablas 1, 2, 3 y 4). Si hemos observado que un alumno/a no ha logrado seleccionar toda la información de un discurso escrito considerada como relevante, es porque ha tenido dificultades a la hora de aplicar alguna o algunas de las operaciones cognitivas que figuran como componentes de las Acciones cognitivas de Subrayar y/o Resumir (Tabla 1). Si un alumno/a tiene dificultades en la ejecución de la operación cognitiva construcción del argumento compartido, el resultado será la elaboración incorrecta de la información supraordinada (título inadecuado, Idea Principal no identificada o mal formulada). Si el referente no ha sido concretado o lo ha sido de forma inadecuada, ello afectará a la selección de la información importante, es decir aquella que además de ser nueva es coherente. La categorización de las ideas como relevantes o como triviales ya no será efectuada en base al criterio de coherencia, sino aplicando otros criterios que carecen de valor instrumental con respecto al objetivo de seleccionar la información necesaria. Pero, además, si el título o la Idea Principal carecen de valor referencial, el resultado será que el alumno/a se verá abocado a establecer relaciones arbitrarias entre el referente y las ideas que lo desarrollan. En una actividad de lectura realizada por un grupo de alumnos/as, el argumento compartido formulado, para el DE-1, fue de la siguiente naturaleza y diversidad: la granja de animales; los animales y los humanos; los animales ayudan al hombre a vivir; los animales en el mundo; los animales una fuente de vida para los hombres, los animales son nuestros esclavos, etc. Fácilmente podemos constatar que un referente de estas características convertirá al proceso de comprensión en una actividad con resultados bastante problemáticos. Algo similar ocurrió cuando el objeto de la actividad realizada fue la lectura del DE-2: el fenómeno de la globalización; el sistema económico de los países; intercambios internacionales; la economía española; balanzas en la economía; factores que influyen en la economía de un país; repercusiones de la economía global en la economía local, etc. Las consecuencias de las dificultades producidas en la ejecución de la segunda operación cognitiva (diferenciar entre información nueva e información dada) pueden ser de una naturaleza muy similar: omisión de información relevante. En las pruebas de evaluación, los alumnos/as omiten información (sus respuestas son calificadas como insuficientes), entre otras causas, porque durante el proceso de aprendizaje no la han categorizado como relevante o, simplemente, no ha sido identificada como información nueva. Un resulta- 
do muy parecido suele producirse cuando las dificultades se generan en la ejecución de la operación cognitiva consistente en suprimir la información trivial. Si una parte de la información suprimida ha sido categorizada indebidamente como trivial, nos encontraremos de nuevo ante un caso de omisión injustificada de información relevante y los conocimientos elaborados serán calificados igualmente como insuficientes. En la lectura del DE-1 se han dado un número importante de casos que solamente han seleccionado dos o tres de las cuatro razones expuestas acerca de las utilidades de los animales. En el DE-2, se dieron dos tipos de resultados concretos. Algunos alumnos/as mencionaron la existencia de cinco subbalanzas, al producirse la confusión entre una información supraordinada (la Balanza de Pagos) y la información subordinada referida a cada una de las subbalanzas. En otros casos, se produjo la no selección de una de las subbalanzas que se citan como componente de la Balanza de Pagos (la subbalanza por cuenta de capital, en la mayoría de los casos; la subbalanza de transferencias, en menor proporción). El proceso completo de evaluación abarcaría el análisis de la totalidad de las operaciones cognitivas (Tablas 1, 2, 3 y 4) que definen la composición de cada una de las diferentes Acciones cognitivas. Dadas las limitaciones de espacio, dejamos para otro trabajo una exposición más detallada de las dificultades que se pueden generar en el componente instrumental o funcional del conocimiento estratégico. La finalidad de incluir este apartado en el artículo ha sido la de sugerir un procedimiento para la evaluación de las dificultades en el proceso de comprensión del LE, ya que su conocimiento puede ser de gran utilidad tanto para el diseño de procesos de intervención de naturaleza preventiva como para la elaboración de adaptaciones curriculares en aquellos casos en los que las necesidades educativas identificadas así lo aconsejen. En definitiva, sugerir la conveniencia y pertinencia de hacer de la actividad de lectura/escritura una forma efectiva y eficaz de aprendizaje autorregulado, caracterizado básicamente por el conocimiento de:

a. Los objetivos que se desean alcanzar.

b. La secuencia organizada de operaciones cognitivas que debemos ejecutar para lograrlos.

c. Las dificultades que se pueden generar en su aplicación práctica.

d. Las estrategias metacognitivas relacionadas con el control y evaluación de la actividad cognitiva realizada. 


\section{Referencias}

Ali, K.S. (1991). Instructional strategies that activate preconceptions. En M. Carretero, M. Pope, R.J. Simons y J.I. Pozo (Eds.), Learning and Instruction. vol. 3, 67-82. New York: Pergamon Press.

Ausubel, D.P. (1976). Psicología Educativa. Un punto de vista cognoscitivo. México: Trillas.

Bartlett, F.C. (1932). Remembering : a study in experimental and social psychology. Cambridge, UK: Cambridge University Press.

Beck, I.L., Omanson, R.C. y McKeown, M.G. (1982). An instructional redesign of reading lessons: effects on comprehension. Reading Research Quarterly, 17, 462-481.

Beltrán, J. (1993). Procesos, estrategias y técnicas de aprendizaje. Madrid: Síntesis.

Beltrán, J. (1998). Las estrategias de aprendizaje. En V. Santiuste y J. Beltrán (Coords.), Dificultades de Aprendizaje, 201-240. Madrid: Síntesis.

Bereiter, C. y Bird, M. (1985). Use of thinking aloud in identification and teaching of reading comprehension strategies. Cognition and Instruction. 2, 131-156.

Bereiter, C. y Scardamalia, M. (1987). The psychology of writen composition. Hillsdale, NJ: Lawrence Erlbaum Associates.

Bimmel, P. (2001). Effects of reading strategy instruction in secondary education. A review of intervention studies. L1-Educational Studies in Language and Literature, 1, 273298.

Braten, I. y Samuelstuen, M.S. (2004). Does the influence of reading purpose on reports of strategic text processing depend on students' topic knowledge? Journal of Educational Psychology. 96(2), 324-336.

Brown, A.L. y Day, J.D. (1983). Macrorules for summarizing texts: the development of expertise. Journal of Verbal Learning and Verbal Behavior, 22, 1-14.

Carrell, P.L., Gajdusek, L. y Wise, T. (1998). Metacognition and EFL/ESL reading. Instructional Science, 26, 97-112.

Carriedo, N. y Alonso-Tapia, J. (1994). ¿Cómo enseñar a comprender un texto? Madrid: Ediciones de la Universidad Autónoma de Madrid.

Carriedo, N. y Alonso-Tapia, J. (1995). Comprehension strategy training in content areas. European Journal of Psychology of Education, X(4), 411-431.

Connor, C.M., Morrison, F.J. y Petrella, J.N. (2004). Effective reading comprehension instruction: examining child X Instruction interactions. Journal of Educational Psychology. 96(4), 682-698. 
de Corte, E., Verschaffel, L. y Van de Ven, A. (2001). Improving text comprehension strategies in upper primary school children: a design experiment. British Journal of Educational Psychology, 71, 531-559.

Elosúa, Ma R., García Madruga, J.A., Gutiérrez, F., Luque, J.L. y Gárate, M. (2002). Effects of an intervention in active strategies for text comprehension and recall. The Spanish Journal of Psychology, 5(2), 90-101.

Englert, C.S., Raphael, T.E., Anderson, L.M., Anthony, H.M. y Stevens, D.D. (1991). Making strategies instruction in regular and special education classrooms. American Educational Research Journal, 28(2), 337-372.

Englert, C.S. y Mariage, T.V. (1991). Making students partners in the comprehension process: organization the reading POSSE. Learning Disabilitiy Quarterly, 14, 123-138.

Escoriza, J. (1996a). Perspectiva sociocultural y procesos de influencia educativa. En R. González, A. Barca, J. Escoriza y J. A. González (Eds.), Psicología de la Instrucción, vol. 1, 171-199. Barcelona: Ediciones Universitarias de Barcelona.

Escoriza, J. (1996b). Psicopedagogía del Lenguaje Escrito: la lectura. En J. Escoriza, J.A. González, R. González y A. Barca (Eds.), Psicología de la Instrucción, Vol. 4. 89-132. Barcelona: Ediciones Universitarias de Barcelona.

Escoriza, J. (1998a). Conocimiento psicológico y conceptualización de las Dificultades de Aprendizaje. Barcelona: Edicions Universitat de Barcelona.

Escoriza, J. (1998b). Dificultades en el proceso de composición del discurso escrito. En V. Santiuste y J. Beltrán (Coords.), Dificultades de Aprendizaje, 147-162. Madrid: Síntesis.

Escoriza, J. (2002). Análisis de las dificultades en la explicitación del conocimiento estratégico durante el proceso de comprensión lectora. Revista Galego-Portuguesa de Psicoloxía e Educación, 6(8), 243-290.

Escoriza, J. (2003). Evaluación del conocimiento de las Estrategias de Comprensión Lectora. Barcelona: Edicions Universitat de Barcelona.

Escoriza, J. (2004a). Fundamentación teórica y descripción de una propuesta de innovación docente universitaria. Revista Galego-Portuguesa de Psicoloxía e Educación, 9(11), $19-57$.

Escoriza, J. (2004b). La actividad de aprendizaje del alumno/a guiada por la aplicación de estrategias para la comprensión y expresión del conocimiento. Tercer Congreso Internacional de Docencia e Innovación Universitaria, organizado por las Universidades 
de Barcelona, Autónoma de Barcelona, Girona y Politécnica de Cataluña. Girona 30 de junio y 1y 2 de julio del 2004.

Escoriza, J. (en prensa). Dificultades en la expresión/comunicación del conocimiento mediante la producción de un discurso escrito. En J. Escoriza (Coord.), Tratamiento educativo de los trastornos de la lengua escrita. Barcelona: Edicions Universitat de Barcelona.

Escoriza, J. y Boj, C. (1997). Psicopedagogía de la escritura. Barcelona: Llibreria Universitaria de Barcelona.

Frederiksen, C.H. y Donin, J. (1991). Constructing multiple semantic representations in comprehending and producing discourse. En G. Denhiere y J.P. Rossi (Eds.), Text and text processing, 19-44. Amsterdam: North Holland.

Gersten, R., Fuchs, L.S., Williams, J.P. y Baker, S. (2001). Teaching reading comprehension strategies to students with learning disabilities: a review of research. Review of Educational Research, 71(2), 279-320.

Graesser, A.C., Millis, K.K. y Zwaan, R.A. (1997). Discourse comprehension. Annual Review of Psychology. 87, 163-189.

Greenway, C. (2002). The process, pitfalls and benefits of implementing a Reciprocal Teaching Intervention to improve the reading comprehension of a group of 6 year pupils. Educational Psychology in Practice, 18(2), 113-137.

Guthrie, J.T., Wigfield, A., Barbosa, P., Perencevich, K.C., Taboada, A., Davis, M.H., Scafiddi, N.T. y Tonks, S. (2004). Increasing reading comprehension and engagement through concept-oriented reading instruction. Journal of Educational Psychology. 96(3), 403-423.

Hansen, J. (1981). The effects of inference training and practice on young children's reading comprehension. Reading Research Quarterly, 3, 391-417.

Hayes, J. y Flower, L. (1980). Identifying the organization of writing processes. En L. Gregg y E. Teinberg (Eds.), Cognitive processes in writing, 3-30. Hillsdale/New York: Erlbaum.

Janssen, T. (2002). Instruction in self-questioning as a literary reading strategy: an exploration of empirical research. L1-Educational Studies in Language and Literature, 2, 95-120.

Johnson-Laird, P.N. (1983). Mental models. Towards a cognitive science of language, inference and consciousness. Cambridge, MA: Harvard University Press.

Just, M.A. y Carpenter, P.A. (1980). A theory of reading: from eye fixations to comprehension. Psychological Review, 87, 329-354. 
Karpov, Y. (1995). L.S. Vygotsky as the founder of a new approach to instruction. School Psychology International, 16(2), 131-142.

Kendeou, P. y van den Broek, P. (2005). The effects of readers' misconceptions on comprehension of scientific text. Journal of Educational Psychology. 97(2), 235-245.

King, J.R., Biggs, S. y Lipsky, S. (1984). Students self-questioning and summarizing and reading study strategies. Journal of Reading Behavior, 16, 205-218.

King, C.M. y Johnson, L.M. (1999). Constructing meaning via Reciprocal Teaching. Reading Research and Instruction Spring, 38(3), 169-186.

Kintsch, W. y van Dijk, T.A. (1978). Toward a model of text comprehension and production. Psychological Review, 85, 363-394.

Kintsch, W. (1988). The role of knowledge in discourse comprehension: a constructionintegration model. Psychological Review, 95(2), 163-182.

Lensky, S.D. y Nierstheimer, S.L. (2002). Strategy instruction from a sociocognitive perspective. Reading Psychology, 23, 127-143.

Leontiev, A.N. (1990). Sign and activity. En J.V. Wertsch (Ed.), The concept of Activity in soviet psychology. New York, M.E: Sharpe.

Lorch, R.F. y van den Broek, P. (1997). Understanding reading comprehension: current and future contributions of cognitive science. Contemporary Educational Psychology, 22, 213-246.

Maria, K. (1987). A new look at comprehension instruction for disabled readers. Annals of Dyslexia, 37, 264-278.

Novak, J.D. (2002). Meaninful learning: the essential factor for conceptual change in limited or inappropriate propositional hierarchies leading to empowerment of learners. Science Education, 86(4), 548-571.

Palincsar, A.S. y Brown, A.L. (1984). Reciprocal Teaching of comprehension-fostering and comprehension-monitoring activities. Cognition and Instruction, 1(2), 117-175.

Paris, S.G., Lipson, M.Y. y Wixson, K. (1983). Becoming a strategic reader. Contemporary Educational Psychology. 8, 293-316.

Pearson, P.D. y Gallagher, M.C. (1983). The instruction of reading comprehension. Contemporary Educational Psychology. 8, 317-344.

Pressley, M. (1998). Comprehension strategies instruction. En J. Osborn y F. Lehr (Eds.), Literacy for all. Issues in teaching and learning, 113-133. New York: The Guilford Press. 
Pressley, M., Johnson, C.J., Symons, S., McGoldrick, J.A. y Kurita, J.A. (1989). Strategies that improve children's memory and comprehension of text. Elementary School Journal, 90, 3-32.

Pressley, M., El-Dinary, P.B., Gaskins, I., Schuder, T., Bergman, J. Almasi, L. y Brown, R. (1992). Beyond direct explanation: transactional instruction of reading comprehension strategies. Elementary School Journal, 92, 511-554.

Pressley, M. y Woloshyn, V. (1995). Cognitive strategy instruction that really improve children's academia perfomance. Cambridge, MA: Brookline Books.

Robinson, F.P. (1946). Effective study. New York: Harper and Row.

Roehler, L.R. y Duffy, G.G. (1984). Direct explanation of comprehension processes. En G.G. Duffy, L. R. Roehler, \& J. Mason (Eds.), Comprehension instruction: perspectives and suggestions, 265-280. New York: Longman.

Rosenshine, B. y Meister, C. (1984). Reciprocal teaching: a review of the research. Review of Educational Research, 64, 450-479.

Sánchez, E. (1989). Procedimientos para instruir en la comprensión de textos. Madrid:CIDE.

Sanders, T. (1992). Discourse structure and coherence. Aspects of a cognitive theory of discourse representation. Unpublished doctoral dissertation. Tilburg University, The Neetherlands.

Sanders, T. y Noordman, L. (2000). The role of coherence relations and their linguistic markers in text processing. Discourse Processes, 29(1), 37-60.

Schirmer, B.R. (2003). Using verbal protocols to identify the reading strategies of students who are deaf. Journal of Deaf Studies and Deaf Education, 8(2), 157-170.

Schraw, G. (1998). Promoting general metacognitive awareness. Instructional Science, 26, 113-125.

Smolkin, L.D. y Donovan, C.A. (2001). The context of comprehension: the information book read aloud, comprehension acquisition, and comprehension instruction in first-grade classroom. The Elementary School Journal, 102(2), 97-122.

Sovik, N., Samuelstuen, M. y Flen, A. (2000). Cognitive and linguistic predictors of text comprehension. European Journal of Psychology of Education, XV(2), 135-155.

Tharp, R.G. (1982). The effective instruction of comprehension: results and description of the Kamehameha Early Education Program. Reading Research Quarterly, 17, 503-527.

Thiede, K.W. y Anderson, M.C.M. (2003). Summarizing can improve metacomprehension accuracy. Contemporary Educational Psychology, 28, 129-160. 
Torgesen, J.K. (1977). Memorization processes in reading disabled children. Journal of Educational Psychology, 69, 571-578.

Torgesen, J.K. (1991). Learning disabilities: historical and conceptual issues. En B. Wong (Ed.), Learning about learning disabilities. Baltimore: University Park Press.

van Dijk, T.A. y Kintsch, W. (1983). Strategies of discourse comprehension. New York: Academia Press.

Vidal-Abarca, E. y Gilabert, R. (1991). Comprender para aprender. Madrid: CEPE.

Vidal-Abarca, E. y Gilabert, R. (1994). Mapas de ideas: una herramienta para el aprendizaje escolar. Datos y comentarios para una discusión. Comunicación, Lenguaje y Educación, 21, 75-86.

Vosniadou, Sh. (1991). Children's naive models and the processing of expository text. En M. Carretero, M. Pope, R.J. Simons y J.I. Pozo (Eds.), Learning and Instruction. vol. 3, 325-336. New York: Pergamon Press.

Willoughby, T., Word, E., McDermott, C. y McLaren, J. (2000). Enhancing learning through strategy instruction and group interaction: is active generation of elaborations critical?. Applied Cognitive Psychology, 14, 19-30.

Willoughby, T., Wood, E. y Kraftcheck, R. (2003). When can a lack of structure facilitate strategic processing of information?. British Journal of Educational Psychology, 73, 59-69.

Winograd, P.N. (1985). Dificultades de estrategia en el resumen de textos. Infancia y Aprendizaje, 31-32, 67-87 


\section{ANEXO}

Discursos escritos empleados para ejemplificar la aplicación práctica de las diferentes operaciones cognitivas que han sido expuestas:

\section{Discurso Escrito 1 (DE-1):}

"Los animales son útiles por muchas razones. Los animales nos dan comida. Por ejemplo, conseguimos carne de las vacas y de los cerdos y huevos de las gallinas.

Los animales son útiles para el trabajo y el transporte. Los caballos llevan a las personas y tiran de las carretas. Las mulas pueden arar los campos y llevar cargas. En algunos países, los elefantes hacen el trabajo pesado que los humanos no podrían hacer.

Conseguimos otros productos de los animales. El cuero que usamos para abrigos, cinturones, monederos y equipos de deporte, procede de las vacas, los cerdos e incluso canguros. El jabón y muchos productos químicos que se emplean en la comida y en la medicina proceden de los animales.

Los animales también nos proporcionan diversión. Cabalgar es muy divertido. Pescar es un deporte que muchos disfrutan y a muchas personas les gusta vivir con perros, gatos, peces y pájaros" (Bauman, 1990, p. 161).

\section{Discurso Escrito 2 (DE-2):}

"Los ciudadanos de los diversos estados realizan una gran variedad de intercambios económicos que se materializan en ingresos y pagos. Las transacciones económicas, llevadas a cabo, requieren la existencia de alguna forma de pago internacional. Dichos pagos se hacen efectivos mediante divisas que son adquiridas por todas aquellas personas físicas y/o jurídicas que las necesitan para comprar en el extranjero, hacer inversiones, etc. El problema es que cada estado tiene su propio sistema monetario y que, debido a su carácter fiduciario e inestable, crea dificultades a la hora de emplearlo para financiar las prestaciones o intercambios que se producen entre los diferentes estados. La contabilidad de las operaciones económicas con el exterior se hace efectiva en la llamada Balanza de Pagos en la que quedan registradas sistemáticamente las transacciones económicas realizadas anualmente entre los residentes de un país y los del resto del mundo.

El valor de las importaciones y de las exportaciones, de bienes materiales, queda registrado en la balanza de mercancías e incluye el precio del producto y los gastos complementarios que se han generado. Si un país compra a otro naranjas, acero, tractores, etc. el valor de estas importaciones se contabiliza como pago. Sin embargo, si lo que hace es vender patatas, queso, trenes, etc. el valor de las exportaciones se contabiliza como ingresos. Los estados también intercambian servicos de todo tipo, tales como seguros, turismo, transporte, etc. quedando registrados en la balanza de servicios. Hay estados que tienen mucho turismo y otros poco, dependiendo de factores muy diversos. Cuando el volumen de turismo es importante, se crean puestos de trabajo que atraen a los trabajadores agrícolas, con el consiguiente abandono del campo y la disminución de la producción en el sector primario. Las zonas rurales pueden quedar desiertas debido al incremento emigratorio hacia las grandes ciudades.

Pero la emigración también puede ser exterior. En este supuesto, los trabajadores/as recibirán un pago por la actividad productiva desarrollada y que, en la mayoría de los casos, suelen enviarlo a sus familiares. Estos envíos de dinero, quedan registrados en la llamada balanza de transferencias. Los ingresos, procedentes de la emigración exterior, han supuesto un evidente beneficio para el desarrollo económico de algunos estados. España ha sido un ejemplo y, en la actualidad, está ocurriendo un fenómeno similar con algunos estados africanos. Ademas, de todos estos intercambios, tenemos que hacer referencia a los préstamos, inversiones, etc. a largo plazo o importaciones/exportaciones de capital. Las empresas multinacionales, las personas físicas, etc. invierten su dinero en otros estados, ya sea para instalar una fábrica, invertir en bolsa, comprarse una vivienda, etc. Todos estos movimientos de capital, quedan registrados en la balanza por cuenta de capital. Hoy día, se habla de la globalización y de sus consecuencias. Como en todas las controversias, 
unos defienden la inversión extranjera y otros están en contra. Lo cierto es que el volumen de inversión exterior, por una parte, influye en la creación de empleo pero, por otra, genera un incremento del control de la economía interna por agentes externos". 